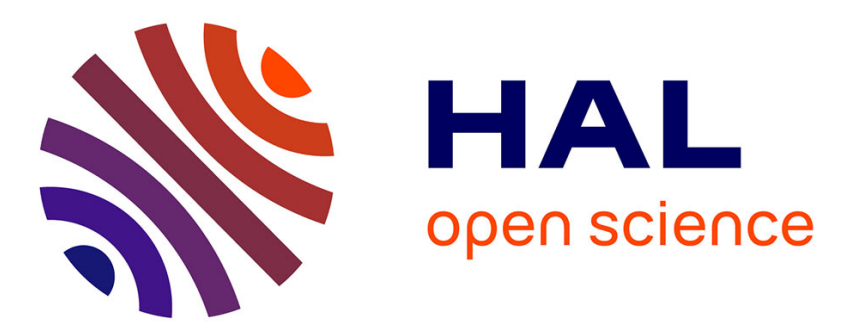

\title{
Exact nonlinear analytic Vlasov-Maxwell tangential equilibria with arbitrary density and temperature profiles
}

Fabrice Mottez

\section{- To cite this version:}

Fabrice Mottez. Exact nonlinear analytic Vlasov-Maxwell tangential equilibria with arbitrary density and temperature profiles. Physics of Fluids, 2003, 10, Phys. Plasmas, 10, pp. 2501-2508. hal00001083

\section{HAL Id: hal-00001083 \\ https://hal.science/hal-00001083}

Submitted on 28 Jan 2004

HAL is a multi-disciplinary open access archive for the deposit and dissemination of scientific research documents, whether they are published or not. The documents may come from teaching and research institutions in France or abroad, or from public or private research centers.
L'archive ouverte pluridisciplinaire HAL, est destinée au dépôt et à la diffusion de documents scientifiques de niveau recherche, publiés ou non, émanant des établissements d'enseignement et de recherche français ou étrangers, des laboratoires publics ou privés. 


\title{
Exact nonlinear analytic Vlasov-Maxwell tangential equilibria with arbitrary density and temperature profiles
}

\author{
F. Mottez, \\ Centre d'étude des Environnements Terrestre et Planétaires (CETP), \\ 10-12 Av. de l'Europe, 78140 Vélizy, France. \\ (e-mail: fabrice.mottez@cetp.ipsl.fr)
}

January 28, 2004

\begin{abstract}
The tangential layers are characterized by a bulk plasma velocity and a magnetic field that are perpendicular to the gradient direction. They have been extensively described in the frame of the MagnetoHydro-Dynamic (MHD) theory. But the MHD theory does not look inside the transition region if the transition has a size of a few ion gyroradii. A series of kinetic tangential equilibria, valid for a collisionless plasma is presented. These equilibria are exact analytical solutions of the Maxwell-Vlasov equations. The particle distribution functions are sums of an infinite number of elementary functions parametrized by a vector potential. Examples of equilibria relevant to space plasmas are shown. A model for the deep and sharp density depletions observed in the auroral zone of the Earth is proposed. Tangential equilibria are also relevant for the study of planetary environments and of remote astrophysical plasmas.

(C) Copyright (2003) American Institute of Physics. This article may be downloaded for personal use only. Any other use requires prior permission of the author and the American Institute of Physics. The following article appeared in Phys. Plasmas, Vol 10, 7, pp2501,2508, and may be found at http://link.aip.org/link/?php/10/2501.
\end{abstract}




\section{INTRODUCTION}

The swedish Viking spacecraft orbiting in the auroral region of the magnetosphere discovered deep plasma density depletions [1]. Their size along the magnetic field direction reach thousands of kilometers. They can be considered as channels parallel to the magnetic field. These observation were confirmed by measurements onboard the Fast Auroral SnapshoT explorer (FAST) spacecraft [2]. The data showed a lot of plasma activity in and around these regions, particularly plasma acceleration (at the origin of the polar auroras) [3], electromagnetic radiation [4] and a high level of electrostatic turbulence. Most of the studies focused on the acceleration [5], [6] and the nature of the turbulence [7], [8] [9]. But surprisingly, little work has been devoted to the structure of these plasma cavities. Some theoretical works have been devoted to their origin, but none has tackled the question : are auroral plasma cavities in equilibrium, or do they vanish a soon as the cause of their formation disapears?

The aim of the work presented in this paper is to construct a kinetic model of the auroral plasma cavities. But as a very general solution to this problem is found, the field of applications appears much wider. I present here a family of equilibrium solutions that can be used in many domains of the physics of plasmas. They will be named as tangential equilibria because, as in the MHD tangential discontinuities, the magnetic field and the bulk velocities are tangential to the plane $(y, z)$ of invariance.

Previous works on tangential equilibria [10], [11] have shown the existence of simple isothermal solutions that can be computed analytically. Other works, mainly focused on the study of the Earth magnetopause [12], have shown solutions that satisfy a larger class of constraints, but they cannot be constructed analytically.

Most of these equilibria a based, for each species, on distribution functions that are products of an exponential function of the total energy and of $p_{z}^{2}$, by an arbitrary $g$ function of $p_{y}$,

$$
f=\left(\frac{\alpha_{z} \alpha_{\perp}^{2}}{\pi^{3}}\right)^{1 / 2}\left[\exp \left(-\alpha_{z} v_{z}^{2}-\alpha_{\perp} v_{\perp}^{2}\right)\right] g\left(m v_{y}+q A_{y}(x)\right),
$$

where $\alpha_{z}=m / 2 T_{z}=1 / v_{t z}^{2}, \alpha_{\perp}=m / 2 T_{\perp}=1 / v_{t \perp}^{2}$, are the reciprocals of the squared thermal velocities ; $v_{z}=p_{z} / m$ and $v_{\perp}^{2}=v_{x}^{2}+v_{y}^{2}$. If $g(\infty) \sim 1$, then $f$ is a bi-maxellian distribution for $x \sim \infty$. 
Krall and Rosenbluth, 13 have derived a solution of the linearized kinetic equations, by setting $g\left(p_{y}\right)$ as a linear function. They used this simple solution for the study of gradient instabilities.

Many authors (reviewed by Roth et al. [12]), who studied tangential discontinuities, considered solutions with $g$ functions that involve error functions. Most of these studies where developed for the study of the equilibrium of the Earth magnetopause. They include an electric field $E_{x}$ and no charge neutrality. There is no analytical solution. The differential equations on $E_{x}$ and $A$ are solved through a numerical integration.

Channell [11] considered solutions with no electric field. He showed that

$$
J_{y}=T_{\perp} \frac{d n}{d A_{y}} .
$$

A more general formula will be shown in Sec. II.

In the same paper, Channell studied a few examples of solutions with simple but nonlinear $g$ functions. In particular, he showed that there exists a solution with $g\left(p_{y}\right)=\exp \left(-\eta p_{y}^{2}\right)$, where $\eta$ is a constant number.

Harris [10] built an equilibrium model of a current sheet. His model was widely used later for the study of the tearing mode instability. The Harris current sheet equilibrium corresponds to the case $g\left(p_{y}\right)=\exp \left(\nu p_{y}\right)$.

All the solutions mentionned above are based on elementary $g$ functions. They do not let the possibility of setting a priori a density profile, as in the more simple MHD and bi-fluid models. We can build a distribution function that is the sum of such elementary distribution functions and assert that each elementary part of the distribution corresponds to a family of trapped particles.

Because of the nonlinearity of the equations, the density and temperature profiles associated to a sum of families of trapped particles are not simple combinations of the profiles associated to single elementary particle distributions. Nethertheless, using a numerical equation solver, Roth et al. 112 added up to four elementary distributions functions (all of them were built with error functions). Differential equations were solved numerically.

I show in this paper how to combine an infinite number of elementary solutions and build a plasma that is made of a continuum of famillies of trapped particles. The plasma equilibria presented in this paper are computed analytically. They do not have the high degree of generality of those shown in Roth et al. [12, (because I impose a null electric field), but they 
are more general than those of Harris [10], and Channell [11]. In particular, the plasma does not have to be isothermal.

The equations of the kinetic model are developed and solved in Sec. II. The properties of the solutions and some particular cases are briefly analysed in Sec. III. An equilibrium model for the deep non isothermal density depletions of the Earth auroral zone is presented. The last section is a discussion about other applications and further developments.

\section{FORMULATION OF THE MAXWELL- VLASOV MODEL}

We consider a monodimensional equilibrium : $\partial_{t}=0$ and $\nabla=\left(d_{x}, 0,0\right)$. We choose the $z$ direction along the constant direction of the magnetic field $\overrightarrow{\mathbf{B}}=\left(0,0, B_{z}(x)\right),(\nabla \cdot B=0$ is therefore ensured $)$. The magnetic field derives from a vector potential $\overrightarrow{\mathbf{A}}(x)=\left(0, A_{y}(x), 0\right)$ such that $B_{z}=d_{x} A_{y}$. This vector potential satisfies the Lorentz gauge (that is $\nabla \cdot \overrightarrow{\mathbf{A}}=0$ in the stationary case). The $x$ direction is called the normal direction, the $y, z$ plane is the tangential plane.

In a monodimensional equilibrium, the particles are confined between two points where $v_{x}=0$. In the case of a null electric field, the size $\Delta x$ of this area is

$$
\Delta x=2 v_{\perp} /<\omega_{c}>
$$

where $<\omega_{c}>=(q / m \Delta x) \int_{x_{1}}^{x_{2}} B_{z}(x)$. Absolutely no restriction on the variations of $B_{x}$ is made.

As we look for a monodimensional equilibrium, the total energy $E=$ $\frac{m v^{2}}{2}+q \Phi$ of any particle and the generalized momentum $p_{y}=m v_{y}+q A_{y}$ and $p_{z}=m v_{z}$ are the invariants of the motion. Any distribution function of the form $f=f\left(E, p_{y}, p_{z}\right)$ is a solution of the Valsov equation. All the kinetic solutions studied in this paper will be of that form. The other character imposed on the solutions is a null electric field, and therefore no charge separation.

The basic idea is to decompose linearly the $g$ function (already mentionned in Eq. 1) over a set of elementary distribution functions that correspond to an analytical equilibrium solution. As will be shown in this section, if the sum is parametrized by a parameter $a$ that correspond to shifts of the 
elementary distribution functions in the space of the potential vectors (and not in the space of the configurations), there is an analytical solution.

For instance, we can show that a continuous linear combination of Channell and Harris-like $g$ distribution functions given by

$$
g\left(p_{y}\right)=n_{0}+\int_{a_{1}}^{a_{2}} d a n_{g}(a) \exp \left[-\eta(a)\left(\frac{p_{y}}{m}-\frac{q}{m} a\right)^{2}+\nu(a)\left(\frac{p_{y}}{m}-\frac{q}{m} a\right)\right],
$$

leads to an equilibrium that can be computed analytically.

The functions $n_{g}(a), \eta(a)>0$, and $\nu(a)$ are defined almost arbitrarily and $n_{0}$ is a constant scalar. Each of these three functions provide a degree of freedom and it is possible, as in MHD and bi-fluid models, to control the plasma density profile. But the distribution of the particles depends on the energy through an exponential factor that does not give any freedom to set the temperature profile. Therefore, instead of using 1 and 4 , let us set :

$$
f=\int_{a_{1}}^{a_{2}} d a\left(\frac{\alpha_{z}(a) \alpha_{\perp}^{2}(a)}{\pi}\right)^{1 / 2} e^{\left(-\alpha_{z}(a)\left(v_{z}-u_{z}\right)^{2}-\alpha_{\perp}(a) v_{\perp}^{2}\right)}\left(n_{0}+g_{a}\left(p_{y}\right)\right)
$$

and

$$
g_{a}\left(p_{y}\right)=n_{g}(a) e^{-\eta(a)\left(\frac{p_{y}}{m}-\frac{q}{m} a\right)^{2}+\nu(a)\left(\frac{p_{y}}{m}-\frac{q}{m} a\right)} .
$$

With these distribution functions, a control of the plasma density profile (through $n_{g}(a)$ ) and of the temperature profile of each species (through $\alpha_{z}(a)$ and $\left.\alpha_{\perp}(a)\right)$ is possible. The parameter $a$ is homogeneous to a vector potential.

In order not to overload the equations, we do not express systematically the dependance of the parameters on $a$ and $x$ hereafter.

To solve the charge neutrality equation $\left(n_{i}=n_{e}\right)$ and the Ampère equation

$$
-\frac{d^{2}}{d x^{2}} A_{y}=\mu_{0} J_{y}
$$

we need to compute the contribution of each species $s$ to the charge density,

$$
n_{s}=\int_{a_{1}}^{a_{2}} d a\left(\frac{\alpha_{\perp}}{\pi}\right)^{1 / 2} \int d v_{y} \exp \left(-\alpha_{\perp} v_{y}^{2}\right) g\left(m v_{y}+q A_{y}(x)\right) .
$$

and to the current density,

$$
\begin{aligned}
J_{y s} & =\int_{a_{1}}^{a_{2}} d a q\left(\frac{\alpha_{\perp}}{\pi}\right)^{1 / 2} \int d v_{y} v_{y} \exp \left(-\alpha_{\perp} v_{y}^{2}\right) g\left(m v_{y}+q A_{y}(x)\right) \\
& =\int_{a_{1}}^{a_{2}} d a q\left(\frac{1}{4 \pi \alpha_{\perp}}\right)^{1 / 2} \int d v_{y} \exp \left(-\alpha_{\perp} v_{y}^{2}\right) g^{\prime}\left(m v_{y}+q A_{y}(x)\right)
\end{aligned}
$$


where $g^{\prime}$ is the derivative of $g$. These charge and current densities can be computed explicitely. We reorder Eq. (5) and (6) to separate the terms that depend on $v_{y}$ and $v_{y}^{2}$,

$$
f=\begin{gathered}
\int_{a_{1}}^{a_{2}} d a \sqrt{\frac{\alpha_{\perp}^{2} \alpha_{z}}{\pi^{3}}} e^{-\alpha_{\perp} v_{x}^{2}-\alpha_{z}\left(v_{z}-u_{z}\right)^{2}} \times \\
\left\{n_{0} e^{-\alpha_{\perp} v_{y}^{2}}+n_{g}(a) e^{-\eta(a)\left[\frac{q}{m}\left(A_{y}(x)-a\right)\right]^{2}+\nu(a) \frac{q}{m}\left(A_{y}(x)-a\right)} e^{-P(a) v_{y}^{2}+2 Q(a) v_{y}}\right\},
\end{gathered}
$$

with

$$
P(a)=\alpha_{\perp}+\eta(a) \quad \text { et } Q(a)=\frac{1}{2} \nu(a)-\eta(a) \frac{q}{m}\left(A_{y}(x)-a\right)
$$

The contribution of each species $s$ to the charge and to the current densities are

$$
n_{s}=n_{0}+\int_{a_{1}}^{a_{2}} d a n_{g} \sqrt{\frac{\alpha_{\perp}}{\pi}} e^{-\eta(q / m)^{2} A_{y}^{2}} S_{0, P, Q}
$$

where $S_{0, P, Q}$ is defined by

$$
S_{0, P, Q}=\int_{-\infty}^{+\infty} d v_{y} e^{\left(-P v_{y}^{2}+2 Q v_{y}\right)}=\sqrt{\frac{\pi}{P}} e^{\left(\frac{Q^{2}}{P}\right)}
$$

and

$$
J_{y s}=\int_{a_{1}}^{a_{2}} d a q n_{g} \sqrt{\frac{\alpha_{\perp}}{\pi}} e^{-\eta(q / m)^{2} A_{y}^{2}} S_{1, P, Q},
$$

where

$$
S_{1, P, Q}=\int_{-\infty}^{+\infty} d v_{y} v_{y} e^{\left(-P v_{y}^{2}+2 Q v_{y}\right)}=\frac{Q}{P} \sqrt{\frac{\pi}{P}} \exp \left(\frac{Q^{2}}{P}\right) .
$$

Hence,

$$
\begin{aligned}
n_{s}= & n_{0}+\int_{a_{1}}^{a_{2}} d a n_{g} \sqrt{\frac{\alpha_{\perp}}{\alpha_{\perp}+\eta}} e^{\frac{\nu^{2}}{4\left(\alpha_{\perp}+\eta\right)}} e^{-\frac{\alpha_{\perp}}{\alpha_{\perp}+\eta}\left[\frac{q}{m}\left(A_{y}-a\right)\right]\left[\eta \frac{q}{m}\left(A_{y}-a\right)-\nu\right]} \\
J_{y s}= & -q \int_{a_{1}}^{a_{2}} d a \frac{n_{g}}{2} \sqrt{\frac{\alpha_{\perp}}{\alpha_{\perp}+\eta}} e^{\frac{\nu^{2}}{4\left(\alpha_{\perp}+\eta\right)}} \frac{1}{\alpha_{\perp}+\eta} \times \\
& {\left[-2 \eta \frac{q}{m}\left(A_{y}-a\right)+\nu\right] e^{-\frac{\alpha_{\perp}}{\alpha_{\perp}+\eta}\left[\frac{q}{m}\left(A_{y}-a\right)\right]\left[\eta \frac{q}{m}\left(A_{y}-a\right)-\nu\right]} . }
\end{aligned}
$$

Let

$$
\begin{aligned}
\xi(a) & =\frac{\alpha_{\perp}(a) \eta(a)}{\alpha_{\perp}(a)+\eta(a)}\left(\frac{q}{m}\right)^{2} \\
\delta(a) & =\frac{\alpha_{\perp}(a) \nu(a)}{\alpha_{\perp}(a)+\eta(a)}\left(\frac{q}{m}\right) \\
N_{0}(a) & =n_{g}(a) \sqrt{\frac{\alpha_{\perp}(a)}{\alpha_{\perp}(a)+\eta(a)}} e^{\frac{\nu^{2}}{4\left(\alpha_{\perp}+\eta\right)}} .
\end{aligned}
$$


The contribution of each particle species to the particle density is:

$$
n_{s}(x)=n_{0}+\int_{a_{1}}^{a_{2}} d a N_{0} e^{-\xi\left(A_{y}-a\right)^{2}+\delta\left(A_{y}-a\right)}
$$

Let us set

$$
n_{a}(x)=N_{0}(a) e^{-\xi(a)\left(A_{y}(x)-a\right)^{2}+\delta(a)\left(A_{y}(x)-a\right)},
$$

then

$$
n_{s}(x)=n_{0}+\int_{a_{1}}^{a_{2}} d a n_{a}(x)
$$

If the following equations are satisfied for each value of $a$,

$$
\begin{array}{r}
\xi(a)_{\text {ions }}=\xi(a)_{\text {electrons }} \\
\delta(a)_{\text {ions }}=\delta(a)_{\text {electrons }} \\
N_{0}(a)_{\text {ions }}=N_{0}(a)_{\text {electrons }}
\end{array}
$$

then, the charge neutrality is satisfied. It is easy to show that one can freely choose the functions $\alpha_{\perp}, \eta, \nu, n_{g}$ that correspond to a particle species, then compute the $\xi, \delta$ and $N_{0}$ functions and find the $\eta, \nu, n_{g}$ functions of the other species that are associated with the same $\xi, \delta$ and $N_{0}$. There is only one restriction: $\eta$ must be positive. If we choose electron parameters, the condition for a positive ion $\eta_{i}$ parameter is, for each value of $a$

$$
\frac{m_{e}}{\eta_{e}(a)}>2\left[\left(\frac{m_{i}}{m_{e}}\right) T_{\perp i}(a)+T_{\perp e}(a)\right]
$$

which requires small enough values of $\eta_{e}(a)$. Noticing that $\eta$ homogeneous to the reciprocal of a squared velocity $\eta=v_{\eta}^{2}$, the above relation writes

$$
v_{\eta}^{2}>\left(\frac{m_{i}}{m_{e}} \frac{T_{i}}{T_{e}}+1\right) v_{t e}^{2}
$$

Let us call $\eta_{c}(a)=v_{c}(a)^{-2}$ the value that corresponds to an equality in Eq. (28) and (29). The case of $\eta_{e}=\eta_{c}$ corresponds to an infinite value of $\eta_{i}$. We shall see in the applications that because of the condition $\eta<\eta_{c}, v_{\eta}$ can reach very high values, and it is necessary to remember that $v_{\eta}$ is not an actual velocity, it does not correspond to a propagation phenomenon.

Let us define

$$
k(a)=-\mu_{0} N_{0}(a)\left(\frac{m_{e}}{\alpha_{\perp e}(a)}+\frac{m_{i}}{\alpha_{\perp i}(a)}\right)=-2 \mu_{0} N_{0}(a)\left(T_{\perp e}(a)+T_{\perp i}(a)\right) .
$$


Negative values of $k(a)$ correspond to a positive value of $n(a)$, and therefore imply an increase of the plasma density. On the contrary, positive values of $k(a)$ induce a reduction of the density. The total current density is the sum of the ion and the electron currents,

$$
J_{y}(x)=-\frac{1}{\mu_{0}} \int_{a_{1}}^{a_{2}} d a \frac{k}{2}\left[-2 \xi\left(A_{y}-a\right)+\delta\right] e^{-\xi\left(A_{y}(x)-a\right)^{2}+\delta\left(A_{y}(x)-a\right)} .
$$

Let us notice that Eq. (22), (30), and (31) imply

$$
J_{y}=\int_{a_{1}}^{a_{2}} d a T_{\perp}(a) \frac{d n_{a}}{d A_{y}} .
$$

The above relation is a generalization of Eq. (2) to non isothermal equilibria. The Ampère equation becomes

$$
\frac{d^{2} A_{y}(x)}{d x^{2}}=\int_{a_{1}}^{a_{2}} d a \frac{k(a)}{2}\left[-2 \xi\left(A_{y}(x)-a\right)+\delta\right] e^{-\xi\left(A_{y}(x)-a\right)^{2}+\delta\left(A_{y}(x)-a\right)} .
$$

We can deduce a first order equation

$$
B_{z}(x)^{2}=\left(\frac{d A_{y}}{d x}\right)^{2}=C+\int_{a_{1}}^{a_{2}} d a k(a) e^{-\xi(a)\left(A_{y}-a\right)^{2}+\delta\left(A_{y}(x)-a\right)} .
$$

The vector potential is the solution of

$$
x=s \int_{A_{y}(0)}^{A_{y}(x)} \frac{d A}{\sqrt{C+\int_{a_{1}}^{a_{2}} d a k(a) \exp \left[-\xi(a)(A-a)^{2}+\delta(a)(A-a)\right]}} .
$$

The sign of the magnetic field is given by $s= \pm 1$, and $C$ is an integration constant. The parameters $k, \xi$, and $\delta$ are arbitrary functions of $a$. The solution has a physical meaning if it is defined for any value of $x$ : the integral in Eq. (35) considered as a function of $A_{y}$ must be able to vary from $-\infty$ to $+\infty$.

Equation (34) can be modified in order to provide an integro-differential equation in $B_{z}(x)$. Let $y$ defined by $A_{y}(y)=a$ be the new integration variable. Then,

$$
B_{z}(x)^{2}=C+\int_{y_{1}}^{y_{2}} d y B_{z}(y) k(y) e^{-\xi(y)\left[\int_{y}^{x} B_{z}(u) d u\right]^{2}+\delta(y)\left[\int_{y}^{x} B_{z}(u) d u\right]} .
$$

The same process applied on Eq. (22) provides

$$
n(x)=n_{0}+\int_{y_{1}}^{y_{2}} d y B_{z}(y) N_{0}(y) e^{-\xi(y)\left[\int_{y}^{x} B_{z}(u) d u\right]^{2}+\delta(y)\left[\int_{y}^{x} B_{z}(u) d u\right]} .
$$

These equations will be used in section III.B to analyse the asymptotic behaviour of the solutions. 


\section{EXAMPLES AND PROPERTIES OF TAN- GENTIAL EQUILIBRIA}

\section{A. Examples of elementary solutions with parameters relevant to space plasmas}

The trivial case of a uniform plasma can be easily recovered with several sets of parameters (including $n_{g} \neq 0$ and $\xi \neq 0$ ). The case of the Harris current sheet [10], reviewed in details in [12], can be recovered with $\xi=0, \nu \neq 0$ and $n(a)=n_{g} \delta_{0}$, where $\delta_{0}$ is the Dirac distribution.

Here are a few examples of equilibria where $\nu=0, n_{g}=n_{c} \delta_{0}$, and where the temperature functions are constant. They correspond to the case $g\left(p_{y}\right)=n_{c} \exp \left(-\eta\left(p_{y} / m\right)^{2}\right)$ already mentionned by Channell 11]. The figure given in Channell's paper to illustrate this case displays the equilibrium of an evanescent plasma. We briefly show in this section a few examples of equilibria of finite size current layers in non evanscent plasmas, in order to emphasize a few interesting properties not discussed in Channell's paper.

Figure 1 shows an example of a structure that is computed with parameters typical of high altitude auroral plasmas : asymptotic magnetic field $\sqrt{C}=3000 \mathrm{nT}, n_{0}=10 \mathrm{~cm}^{-3}, T_{i}=T_{e}=10 \mathrm{eV}$, and $\eta_{e}=1.5310^{-16}(\mathrm{~m} / \mathrm{s})^{-2}=$ $0.99 \eta_{c}$, very close to the critical value imposed by Eq. (28). A high negative value $n_{c}=-8 \mathrm{~cm}^{-3}$ allows for a deep density depletion. The abcsissa is normalized to the ion Larmor radius which is $\rho_{L}=152 \mathrm{~m}$. We can see on Fig. 1 that the size of the structure is about $4 \rho_{L} \sim 600 \mathrm{~m}$. Such a narrow and deep structure (the density in the cavity is only $20 \%$ of the density of the surrounding plasma) can be used in a first approximation for the density depletions encountered in the high altitude auroral zone.

The solution displayed in Fig. 2 corresponds to the limiting case for $\eta$, obtained for the weakly magnetized interplanetary plasma encountered in the solar wind at one astronomical unit : $\sqrt{C}=5 \mathrm{nT}, n_{0}=10 \mathrm{~cm}^{-3}$, $T_{i}=T_{e}=100 \mathrm{eV}$, and $\eta_{e}=1.5310^{-17}(\mathrm{~m} / \mathrm{s})^{-2}=0.99 \eta_{c}$. The ion Larmor radius is $280 \mathrm{~km}$. We can notice that for the same density depletion, the variation of the magnetic field is much higher than in the case of a higly magnetized plasma. We also notice that the total size of the structure hardly exceed one ion Larmor radius.

As the size is of the order of the ion Larmor radius, such solutions cannot be described through the fluid theories. Of course, we can also build very 
large density structures, if we choose a small value of $\eta$ in order to get a small value of $\xi$. Such large structures can more simply be described by fluid models. Figure 3 shows the solution with $\eta=10^{-20}(\mathrm{~m} / \mathrm{s})^{-2} \sim 10^{-3} \eta_{c}$. The structure is much larger but has the same amplitude. Comparing Fig. 2 and Fig. 3, we can notice that, contrary to the case of solitons, there is no correlation between the size and the amplitude of the structures.

\section{B. Asymptotic behaviour of the solutions}

We can caracterize two kinds of non trivial elementary solutions : those with $\eta \neq 0$, and those where $\eta=0$ (and therefore $\nu \neq 0$, otherwise the solution is trivial). Those of the first kind correspond to localized solutions, they are centered around an abscissa $x$ such as $A_{y}(x) \sim a$. Therefore, when the distribution is the infinite sum of elementary functions indexed by $a$ as in Eq. (24), the parameter $a$ indicates where the density $n_{a}(x)$ is non negligible. Let us consider an equilibria where, for $x \sim \infty$, the magnetic field has a finite value. Then, the potential vector goes to infinity. Taking $a_{1} \sim-\infty$ and $a_{2} \sim+\infty$ means that the plasma distribution will be influenced by some of those elementary functions even when $x \sim \infty$. On the contrary, equilibria defined with finite values of $a_{1}$ and $a_{2}$ (and $B(\infty) \neq 0$ ) means that for $x \sim \infty$, the equilibria will converge toward the trivial solution of a uniform plasma with the density $n_{0}$ that appears in Eq. (5).

The case of a null magnetic field at $x \sim \infty$ (less useful for space plasmas applications) is different. Let us consider the case of $B(+\infty)=0$. For $x \sim+\infty$, the vector potential has a constant value. Let us call $A_{\infty}$ this value. Then, as $A$ cannot exceed $A_{\infty}$, the main integral in Eq. (35) is divergent for $A_{y}(x)=A_{\infty}$ (otherwise the equilibria would not be defined for any value of $x)$. As the elementary solutions parametrized by $a$ are non negligible only for abscissas $x$ such that $A_{y}(x)$ is close to $a$, it is not necessary to consider values or $a_{2}$ that exceed notably $A_{\infty}$ : they would have no influence on the value of the integral in Eq. (35). In brief, for equilibria with $B(\infty)=0$, the range of values where the parameter $a$ is significant is bounded, $a_{1}$ and $a_{2}$ do not have to be infinite.

In the Harris model, the magnetic field reverses and has two opposite finite values for $x \sim-\infty$ and $x \sim+\infty$. We shall see that there exist non elementary solutions where the magnetic field has finite values on both sides, and that these values have not necessarily the same absolute value.

We consider the case $a_{1}=-\infty$ and $a_{2}=+\infty$. Let $u^{-}=\lim _{x \rightarrow-\infty} u$ be 
the limit of any physical value $u$ when $x$ tends to $-\infty$, and $u^{+}=\lim _{x \rightarrow+\infty} u$. The integral in Eq. (36), where $y_{1}=-\infty$ and $y_{2}=+\infty$ can be cut in three parts : an integral $I 1$ from $y_{1}$ to $x-\Lambda$, an integral $I 2$ from $x-\Lambda$ to $x+\Lambda$, and an integral $I 3$ from $x+\Lambda$ to $y_{2}$. We will consider large values of $x$ (positive or negative), and large values of $\Lambda$.

For $\Lambda$ large enough, $y$ has large negative values,

$$
\begin{aligned}
I 1 & \sim \int_{y_{1}}^{x-\Lambda} d y B_{z}^{-} k^{-} \exp \left(-\xi^{-}\left(B_{z}^{-}\right)^{2}(x-y)^{2}-\delta^{-} B_{z}^{-}(x-y)\right) \\
& \sim-\frac{k^{-}}{\delta^{-}}\left[\exp \delta^{-} B^{-} \Lambda-\exp \delta^{-} B^{-} y_{1}\right]
\end{aligned}
$$

This function tends to zero for $y_{1} \rightarrow-\infty$ if $\delta^{-} B^{-}<0$, and diverges if $\delta^{-} B^{-}>0$. In the case $\delta^{-}=0$,

$$
I 1 \sim \int_{y_{1}}^{x-\Lambda} d y B_{z}^{-} k^{-} \exp \left(-\xi^{-}\left(B_{z}^{-}\right)^{2}(x-y)^{2}\right)
$$

tends toward zero. Similarily, $\lim _{y_{1} \rightarrow-\infty} I 3=0$ if $\delta^{+} B^{+} \geq 0$. With the same kind of technique, we can show that, for large negative values of $x$,

$$
I 2 \sim k^{-} \sqrt{\frac{\pi}{\xi^{-}}} \sigma_{B_{z}^{-}} \exp \frac{\left(\delta^{-}\right)^{2}}{4 \xi^{-}}
$$

where $\sigma_{B_{z}^{-}}$is the sign of $B_{z}^{-}$. Provided that $\delta^{+} B^{+} \geq 0$, and $\delta^{-} B^{-} \leq 0$, the asymptotic expansion of Eq. (36) shows that

$$
\left(B_{z}^{-}\right)^{2}=C+k^{-} \sqrt{\frac{\pi}{\xi^{-}}} \sigma_{\left(B_{z}^{-}\right)} \exp \frac{\left(\delta^{-}\right)^{2}}{4 \xi^{-}} .
$$

Computing an asymptotic value of $I 2$ for $x \rightarrow+\infty$ bring a similar relation for $\left(B_{z}^{+}\right)$. The same method used with Eq. (37) provides the asymptotic value of the contribution of each species to the particle density:

$$
n^{+}=n_{0}+N_{0}^{+} \sigma_{\left(B_{z}^{+}\right)} \sqrt{\frac{\pi}{\xi^{+}}} \exp \frac{\left(\delta^{+}\right)^{2}}{4 \xi^{+}} .
$$

The equation for $n^{-}$is analogous. As $d_{x} n(\infty)=0$, we deduce $J_{y}^{+}=J_{y}^{-}=0$. There is no current density carried by the plasma for $x \sim \infty$. This result can also be found with an asymptotic expansion of Eq. (31). Moreover, Eq. (31) shows that the ion and electron contribution to the current density have the same sign, their mean velocities are therefore opposite. As there is no current for $x \sim \infty$, for each species $v^{+}=v^{-}=0$. The plasma does not flow perpendicularly to the magnetic field at $x \sim \infty$. 


\section{Examples of solutions with and without field reversal}

Figure 4 shows an example of equilibrium where the magnetic field amplitude and the density are not the same for $x \sim-\infty$ and $x \sim+\infty$. We have set constant values $\eta=1.5310^{-17}(\mathrm{~m} / \mathrm{s})^{-2}$ close to $\eta_{c}, \nu=0$, and a function $n_{g}(a)=640$ atanh(1000a). The temperature is $10 \mathrm{eV}$. With $C=(5 n T)^{2}$, the asymptotic magnetic field amplitude is $5 \mathrm{nT}$. The ion Larmor radius is $\rho_{i} \sim 0.210^{6} \mathrm{~m}$. The variable $a$ is homogeneous to $B_{z}$ times $x$, therefore $1000 a \sim x / \rho_{i}$ is of the order of magnitude of the absissa $x$ divided by the ion Larmor radius. The factor 640 that multiplies the inverse hyberbolic tangent function was set heuristically in order to get a variation of density of the order of $60 \%$. The relatively low value of $\sqrt{C}=5 \mathrm{nT}$ allows for high relative variations of the magnetic field amplitude. The asymptotic values of the magnetic field and of the density fit the analytical formulas given in section III.B.

Figure 5 shows an example where $\eta=0$. We have chosen a function $n_{g}(a)=4200 e^{-(1000 a)^{2}}$. The temperature is $10 \mathrm{eV}$, and $\sqrt{C}=5 \mathrm{nT}$. The value $\nu=10^{-10}$ was chosen, because, in the exponential, the factor $\nu a \sim \nu B_{z} x$ is again of the order of $x / \rho_{i}$. We can see on Fig. 5 that the sign of the magnetic field changes for $x \sim-\infty$ and $x \sim+\infty$. The reversal occur for a value of $A_{y}$ that cancels the square root in Eq. (35). Although the magnetic field is reversed on both sides, this is not a Harris equilibrium.

\section{Example of a non isothermal plasma cavity in the Earth auroral zone}

Are the cavities built in the Earth auroral zone in an equilibrium state of the plasma?

Hilgers et al. [1] made a careful analysis of the Langmuir probe current measurements onboard the swedish Viking satellite in low plasma densities. They showed a case, taken at an altitude of $7000 \mathrm{~km}$, of a deep auroral cavity. The density reach less than one particle per cubic centimeter, that is less than $10 \%$ of the surrounding plasma density. The electron plasma temperature in the cavity (although not precisely measured) is of the order of $1 \mathrm{keV}$, compared to $1 \mathrm{eV}$ outside the cavity. The boundaries of the cavity are sharp, Hilgers et al. measured $1.4 \mathrm{~km}$, of the order of a few ion Larmor radii. The magnetic field amplitude is $B_{z 0}=6800 \mathrm{nT}$.

Apart from the temperature gradient, all these features have been qual- 
itatively reproduced in the elementary equilibria shown in Fig. 1. In order to reproduce a cavity with sharp temperature gradients, an elementary solution cannot be used. Let $\rho_{i}$ be the Larmor radius outside the cavity, it corresponds to the cold palsma $(1 \mathrm{eV})$, its value is $21 \mathrm{~m}$. Inside the cavity, the plasma temperature is $1 \mathrm{keV}$, and the ion Larmor radius is $\rho_{i, h o t}=660 \mathrm{~m}=32 \rho_{i}$. We choose an electron temperature function $T_{e}(a)=1+1000 \exp \left(-\left(a / B_{z 0} 32 \rho_{i}\right)^{2}\right)$ that varies from 1 to $1000 \mathrm{eV}$ on a scale $\Delta x \sim 32 \rho_{i} \sim \rho_{i, h o t}$. The density is controled through the constant scalar $n_{0}=10 \mathrm{~cm}^{-3}$, and the $n_{g}$ function, $n_{g}(a)=-n_{g 0} \exp \left(-\left(a / B_{z 0} 32 \rho_{i}\right)^{2}\right)$. The scalar $n_{g 0}$ is set (heuristically) to 50000 in order to have one particle per cubic centimeter inside the cavity. The $\nu$ function is null. The $\eta$ function is chosen is order to get a sharp cavity, that is with a value close to the $\eta_{c}$ function: $\eta(a)=0,98 \eta_{c}(a)$ have values of the order of $10^{-15}(\mathrm{~m} / \mathrm{s})^{-2}$. Actually, the equilibria is not very sensitive on the value of $\eta$. Taking a lower value like $\eta(a)=0.7 \eta_{c}(a)$ (and a slightly lower value of $n_{g 0}$ ) bring similar results.

The result, displayed on Fig. 6, is in quantitative agreement with the prescriptions given by the observations. The size of the gradient is $70 \rho_{i}=$ $1400 \mathrm{~m}$, as measured by Hilgers et al.

We can notice on figure 6 that the magnetic field amplitude variation $\Delta B$ in the cavity is about $0.5 \mathrm{nT}$. A similar equilibria (not displayed) set with a lower value of the ambient magnetic field $\left(B_{z 0}=300 \mathrm{nT}\right)$ bring a larger variation: $\Delta B=5 \mathrm{nT}$.

We conclude from this short study that deep plasma cavities can be equilibrium structures of the Earth auroral plasma. Therefore, they won't be destroyed immediately after the extinction of their cause. It is however interesting to know if this equilibrium is stable. The theoretical treatement of this question goes beyhond the scope of this paper. I will only give a few hints in favour of the stability of the auroral cavities.

The Auroral zone of the Earth is a radio source. The power of the emissions, called Auroral Kilometric Radiation (AKR), can reach 10 MW. The Viking spacecraft has gone through the sources of AKR: the waves are emitted inside the cavities, where they take their free energy from the hot rarefied plasma [7]. The waves are strongly refracted at the edges of the AKR source, and it is probable that a part of the AKR is guided inside the cavities [14]. Auroral kilometric radiations can be observed on time scales of 10 minutes. The cavities that contain AKR sources are expected to last at least for the same duration. Therefore, two situations are possible: (1) the cavities are the consequence of a phenomena that lasts for tens of minutes, or (2) they 
are generated by more transient phenomena and they are stable.

An another hint about the stability of hot plasma cavities comes from numerical simulations carried by Genot et al. [9]. The simulations start with an isothermal (a few eV) auroral cavity. The cavity is stable. An Alfvén wave is added. The interaction of the Alfvén wave with the cavity triggers strong electron acceleration and turbulence. When the free energy is completely removed from the Alfvén wave by the accelerated electrons, the plasma inside the cavity is hot, and the surrounding plasma is cold. We are in the situation shown in Fig 6. The simulations show that the heated cavity, which is still very deep and sharp, is stable.

Most of the models about the generation of auroral cavities invoke strong kinetic Alfvén Waves (SKAW) [15] [16], observed onboard the Freja and FAST satellites [17], [18]. The associated magnetic field perturbation $\Delta B$ is of the order of $50 \mathrm{nT}$. But the observations made onboard FAST show that the SKAW's are mainly observed in the cusp and the polar cap boundary layer, while the cavities are observed in the lower latitude auroral region where the magnetic fluctuations $\Delta B$ do not exceed $5 \mathrm{nT}$. It is possible that the cavities are built in the polar cap boundary layer by SKAWs, they subsist after the disapearance of the Alfvénic perturbations and finally they are gently convected to lower latitudes by the large scale convection electric field. This suppose of course the stability of the auroral cavities. An other scenario is that the cavities are created through Field Line Resonance (FLR) 19 directly in the auroral region. The $\Delta B \sim 5 \mathrm{nT}$ (on a time scale that corresponds to the crossing of a cavity by a spacecraft) associated to the FLR is compatible with the value shown in Fig. 6. The equilibria shown in Fig. 6 may be a relevant short scale Vlasov-Maxwell description of the part of the FLR that is in the high altitude auroral zone (the FLR has been modeled on a global scale in the multifluid approximation up to now).

\section{CONCLUSION AND FURTHER DEVLOPP- MENTS}

This paper presents a large class of monodimensional analytic kinetic equilibria. They are based on particle distribution functions Eq. (5) that depend on a set of almost arbitrary functions. These distribution functions are solutions of the Vlasov equation, and the vector potential can be computed 
through the evaluation of the integral function given in Eq. (35). In some particular cases, like the uniform plasma and the Harris current sheet, this integral is a combination of elementary mathematical functions. In the other cases, its numerical evaluation is straightforward.

Unlike most of the kinetic tangential equilibria given in the litterature, the freedom in the choice of the density profile, altough more difficult to control, is almost as large as with bi-fluid models. The kinetic equilibria have the great advantage of giving a complete description of the distribution function (not provided with the fluid theory), and can describe equilibria where strong gradients develop on the scale of a few ion Larmor radii. These solutions can be used as initial conditions in particle in cell and Vlasov numerical simulations.

Most of the authors who build models and are cited in this paper have based their elementary solutions on distribution functions that depend on the invariants of the motion. Some of them combined a small number of such elementary solutions and asserted that each of these elementary solutions correspond to a family of trapped particles. The distribution function given in Eq. (5) is the superposition, not of a finite number, but of a continuum of families of trapped particles. This is why we have a very large degree of freedom in the choice of the density and temperature profiles. For the first time, we show that such a supperposition of families of trapped particles lead to an analytically integrable equation, whose solution is given in Eq. (35)

The equilibria discussed in section III.B where the plasma is uniform for $x \sim \infty$ can be compared to the solutions of the jump equations developed in the frame of the MHD theory. The equilibria developed in the present paper are characterized by jumps of the density and of the magnetic field, the normal velocity is null as well as the normal magnetic field. They therefore belong to the family of tangential discontinuities. But they concern only a sub-category: the magnetic field direction is uniform and the solutions have no velocity shear: the velocities are equal to zero at $x \sim-\infty$ and $x \sim+\infty$, even if they can take other values at finite distances. These restrictions are not required for general tangential discontinuities.

Building a kinetic model, inspired from the present results, of a tangential discontinuity where the magnetic field can turn is straighforward and will be presented in a forthcomming paper in order to analyse experimental data provided by the Cluster satellites.

A kinetic model of a tangential equilibria whith a velocity shift $(x \sim-\infty$ and $x \sim+\infty$ ) is not compatible with exact charge neutrality. A quasi neutral 
model would do. It could be computed, as a first order perturbation in $n_{i}-n_{e}$ added to the charge neutral equilibria presented in this paper.

The examples given in the present paper come from the magnetospheric and solar wind physics because these media offers the opportunity of in situ observations of non collisional astrophysical plasmas. Tangential equilibria can be used to describe the magnetopause (present around all the magnetized planets with an atmosphere), the distant neutral sheet, the Earth auroral cavities, and some density fluctuations of the solar wind [20].

But tangential equilibria do not exist only in the terrestrial environnment. They exist whenever plasmas with different origins and velocities meet. Such situations exist in many astrophysical plasmas. Theoretical models (based on MHD and plasma multifluid theories) of the boundary of the Heliosphere show that it is constituted of a termination shock, followed by the heliopause, that is a tangential discontinuity, an hydrogen wall, and possibly an heliospheric shock [21]. Tangential discontinuities also exist around other stars. Müller et al. [22] studied the interaction of the very active binary star $\lambda$ Andromedae, and of the neaby star $\epsilon$ Indi with the interstellar medium. They found, as in the case of the heliopause, the existence of four boundaries, one of them, the asteropause being a tangential discontinuity. It is clear that tangential layers exist in other astrophysical plasmas where they may play a very important role, as frontiers, or in acceleration, heating and radiative processes.

The studies of the magnetospheric plasmas have shown that most of the heating and acceleration phenomena can be only explained through kinetic processes. So far, most of the remote astrophysical plasmas have been studied through MHD or multifluid theories. The understanding of acceleration processes in remote astrophysical plasma might as well require kinetic models. The equilibria presented in this paper might be a good start for some of those studies. 


\section{ACKNOWLEDGMENTS}

The author gratefully acknowledges stimulating and usefull discussions with Gérard Belmont and Alain Roux. 


\section{References}

[1] Hilgers, A.,B. Holback, G. Holmgren, and R. Boström, J. Geophys. Res. 97, A6, 8631, (1992).

[2] Strangeway R.J., L. Kepko, R.C. Elphic, C.W. Carlson, R.E. Ergun, J.P. McFadden, W.J. Peria, G.T.Delory, C.C.Chaston, M. Temerin, C.A. Cattell, E. Möbius,L.M. Kistler, D.M. Klumpar, W.K. Peterson, E.G. Shelley, and R. Pfaff, Geosphys. Res. Let., 25, 12, 2065, (1998).

[3] J.P. McFadden, C.W. Carson, R.E. Ergun, C.C. Chaston, F.S. Mozer, M. Temerin, D. M. Klumpar, E.G. Shelley, W.K. Peterson, E. Moebius, L. Kistler, R. Elphic, R. Strangeway, C. Cattell, R. Pfaff Geophys. Res. Lett., 25, 12, 2045, (1998).

[4] Hilgers, A., Geophys. Res. Lett., 19 3, 237-240, (1992).

[5] Génot, V., P. Louarn, and D. Le Quéau, A study of the propagation of Alfvén J. Geophys. Res. 104 22,649, (1999).

[6] V. Génot, P. Louarn, and F. Mottez, JJ. Geophys. Res. 105, A12, 27611, (2000).

[7] A. Roux, A. Hilgers, H de Feraudy, D. Le Queau, P. Louarn, S. Perraut, A. Bahnsen, M. Jesperen, E. Ungstrup, M. Andre, J. Geophys. Res., 98, 11657, (1993).

[8] R. Pottelette, R.A. Treumann, M. Berthomier, J. Geophys. Res., 106, 8465, (2001).

[9] V. Génot, P. Louarn, and F. Mottez, J. Geophys. Res. 106, A12, 29633, (2001).

[10] E.G. Harris, Il Nuovo Cimento 23 1, 115, (1962).

[11] P. J. Channell, Phys. Fluids 19 10, 1541, (1976).

[12] M. Roth, J. De Keyser, M.M. Kuznetova, Space Sci. Rev., 76, 251, (1996).

[13] N.A. Krall and M.N. Rosenbluth, Phys. Fluids 19 2, 254, (1963). 
[14] de Feraudy,H., B.M. Pedersen, A. Bahnsen, and M. Jespersen, Geophys. Res. Lett., 14 511, 1987.

[15] Wu,D.J., D.Y. Wang, and G.L. Huang, Phys. Plasmas, 4 (3), 611, (1997).

[16] Shukla, P.K., L. Stenflo, R. Bingham, Phys. of Fluids, 6, 5, 1677, (1999).

[17] Louarn, P., J.E. Wahlund,T. Chust, H de Feraudy and A. Roux, B. Holback, P.O. Dovner, A.I. Eriksson and G. Holmgren, Geophys. Res. Lett., 21, 17, 1847, (1994).

[18] Chaston, C.C., C.W. Carlson, W.J. Peria, R.E. Ergun and J.P. Mc Fadden, Geophys. Res. Lett. 26,6, 647, (1999).

[19] Lotko,W., A.V. Streltsov, C.W. Carlson, Geophys. Res. Lett., 25, 24, 4449, (1998).

[20] Safrankova, J., Prech, L., Nemecek, Z., Sibeck, D.G. Mukai, T, J. Geophys. Res., 105, 11, 25113, (2000).

[21] Zank, G.P., Space Sci. Rev., 89, 413, (1999).

[22] Müller, H.R., G.P. Zank, and B.E. Wood, Astrophys. J.551, 495, (2001). 

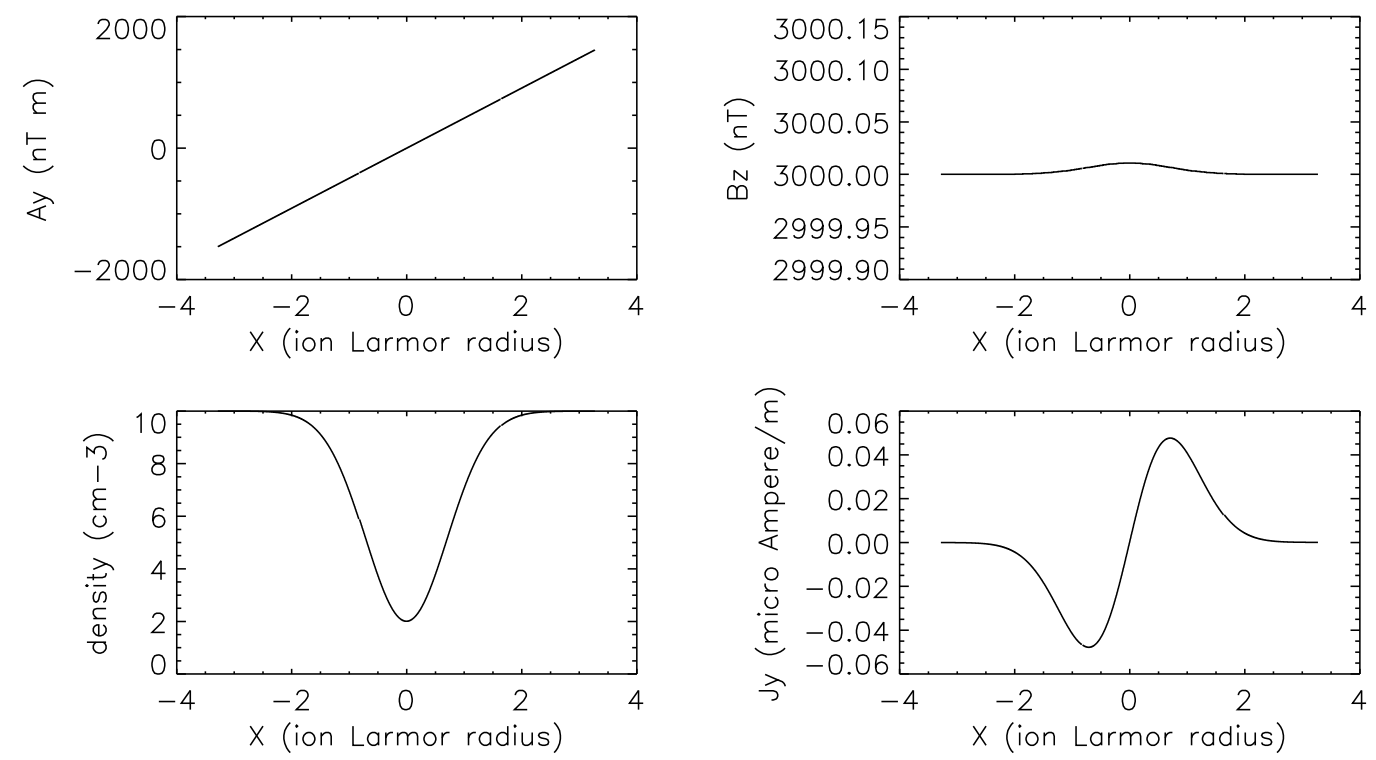

Figure 1: FIG. 1. A deep and narrow density depletion in a highly magnetized plasma. The parameters of this equilibrium are given in Sec. III.A. 

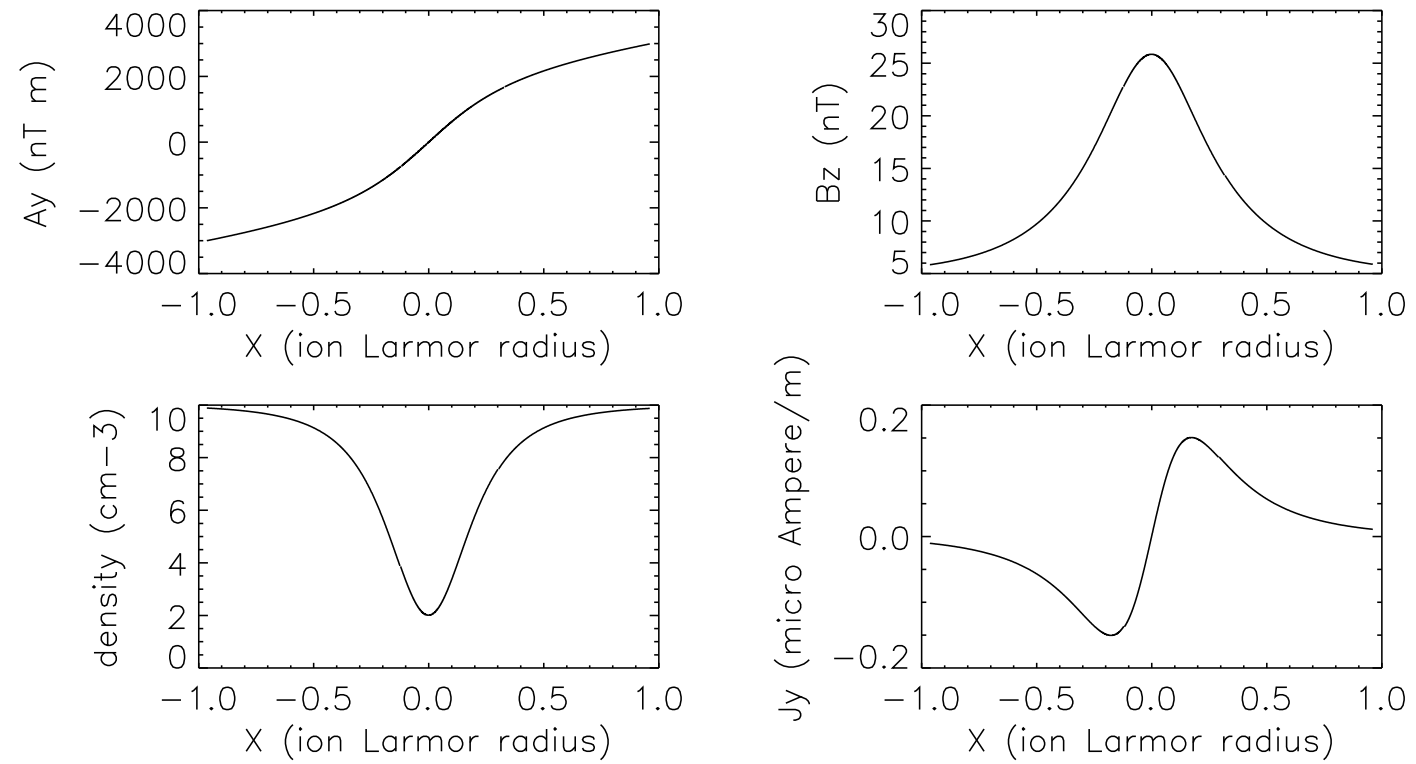

Figure 2: FIG. 2. A deep and narrow density depletion plasma with a weak magnetic field. The parameters are the same as for Fig. 1, exepct for $C$ that is much weaker. 

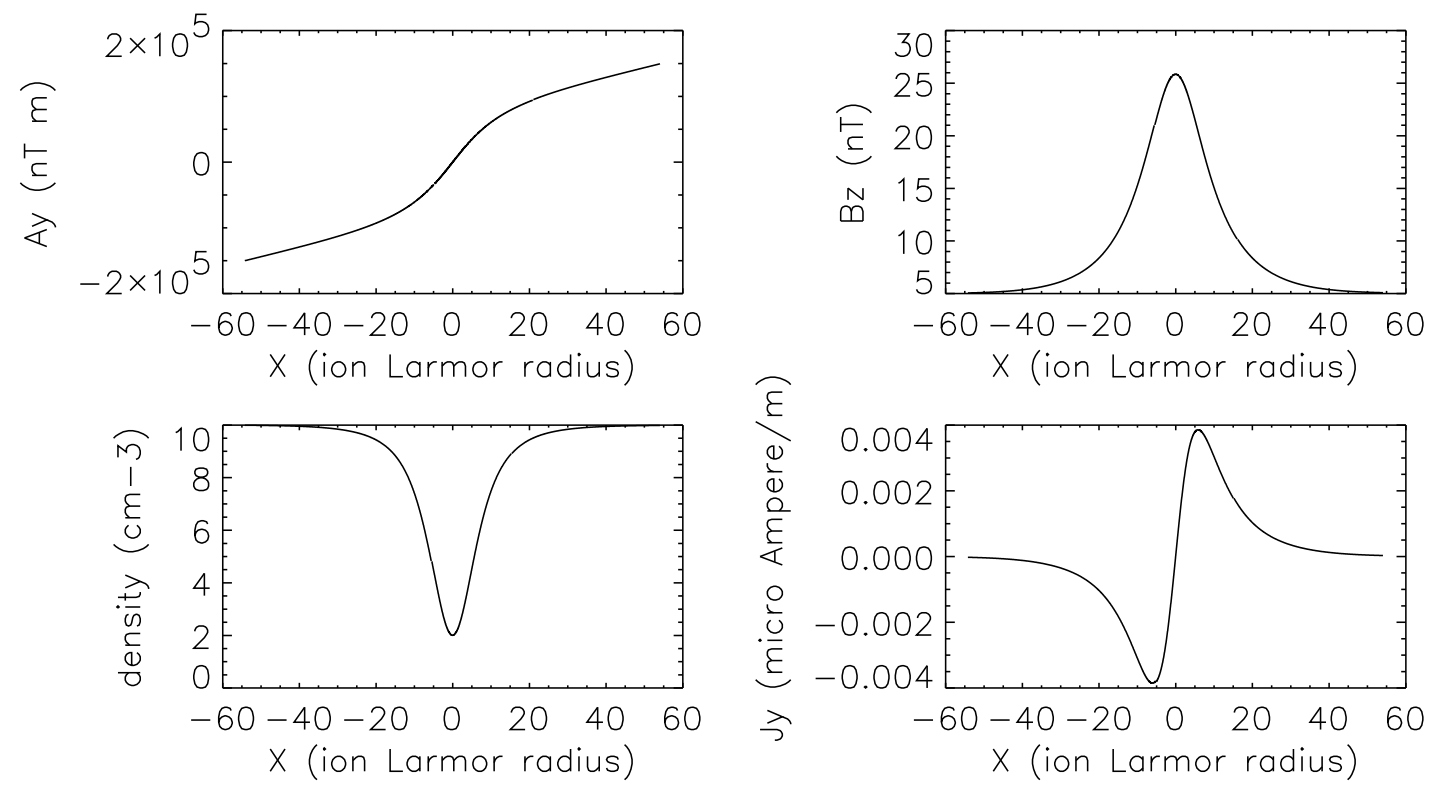

Figure 3: FIG. 3. A deep and large density depletion, the only difference with Fig. 2 is a larger value of $\eta$. See Sec. III.A.. 

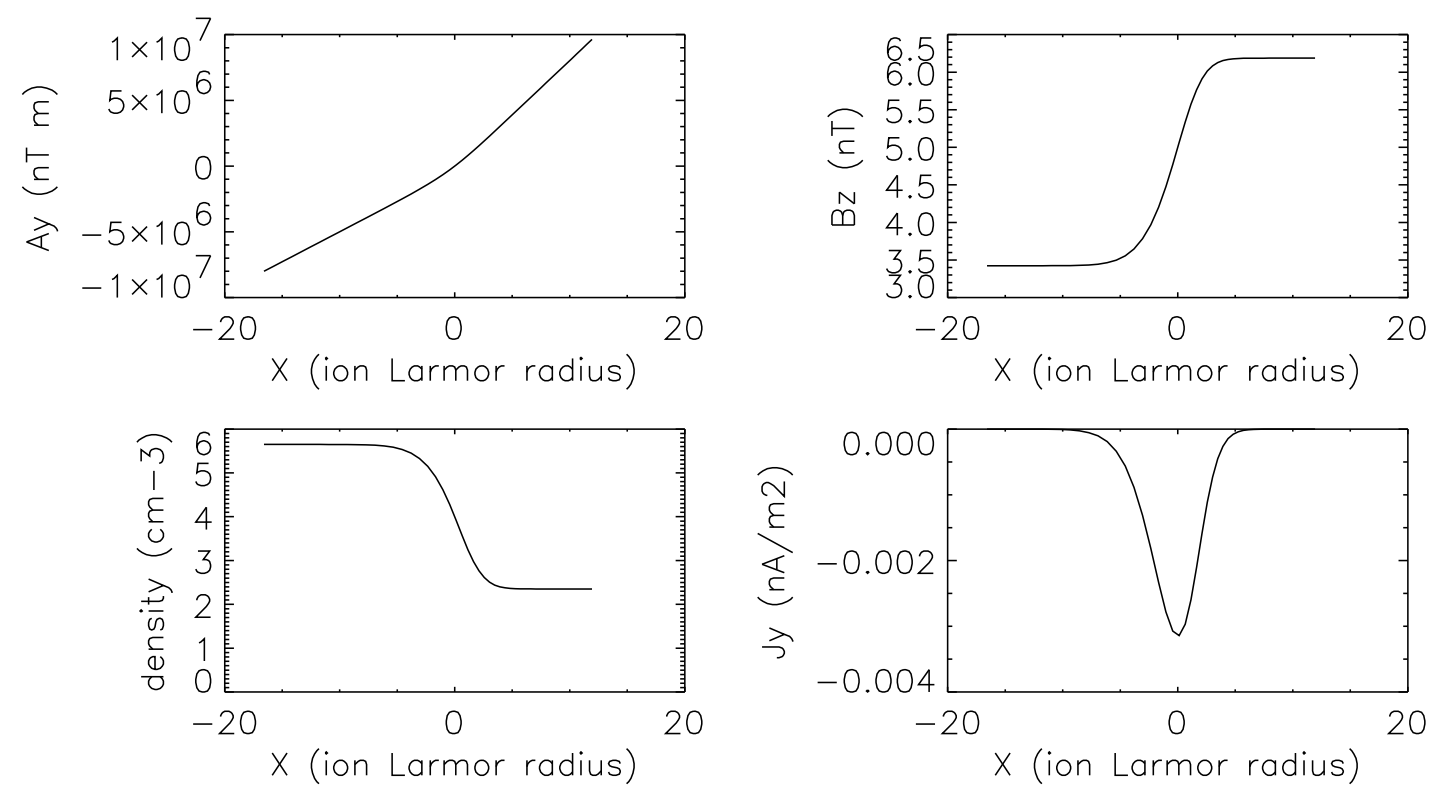

Figure 4: FIG. 4. An example of non symetric equilibrium where $n_{g}$ is a function of $a$. See Sec. III.C for details. 

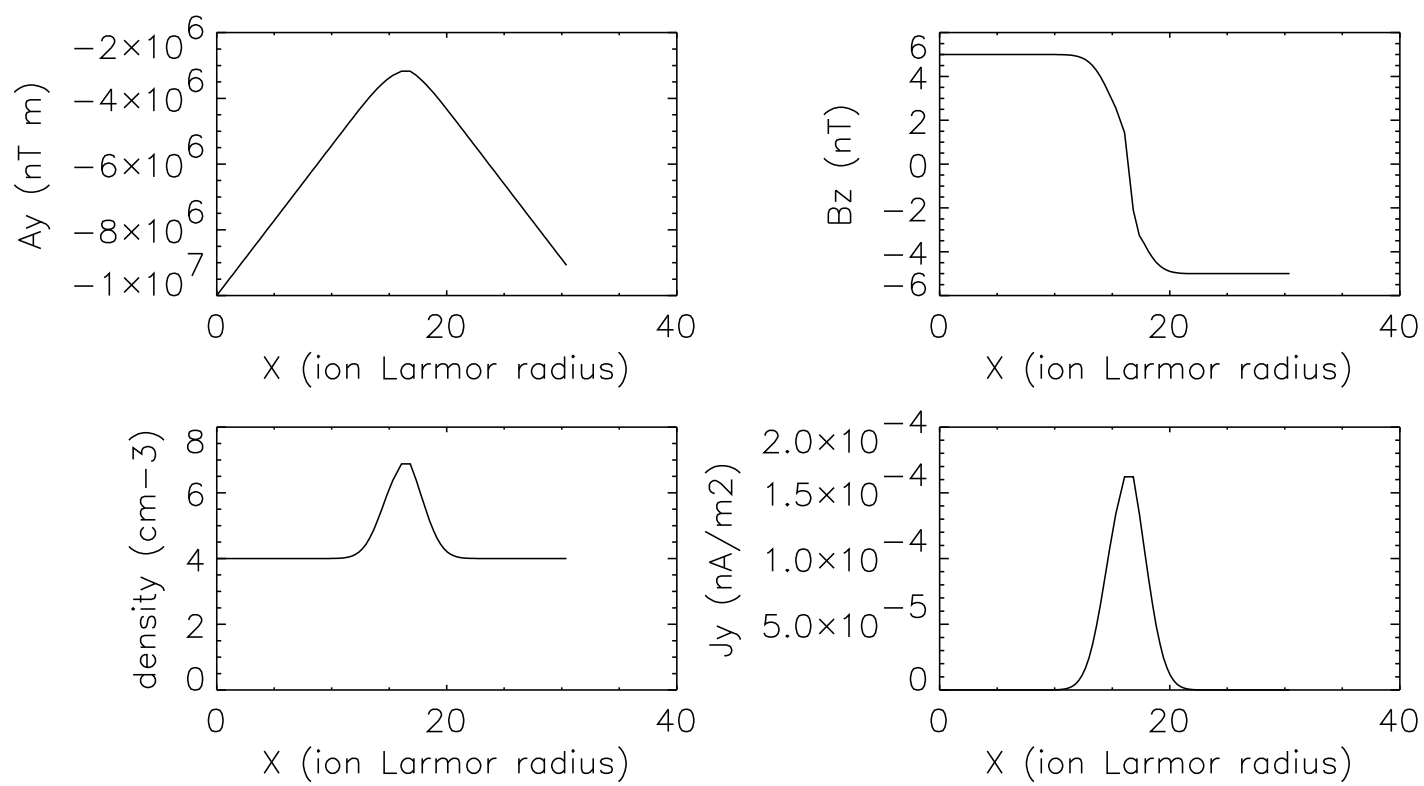

Figure 5: FIG. 5. An exemple of an equilibrium with $\eta=0$ and a reversal of the magnetic field. Details are given in Sec III.C. 

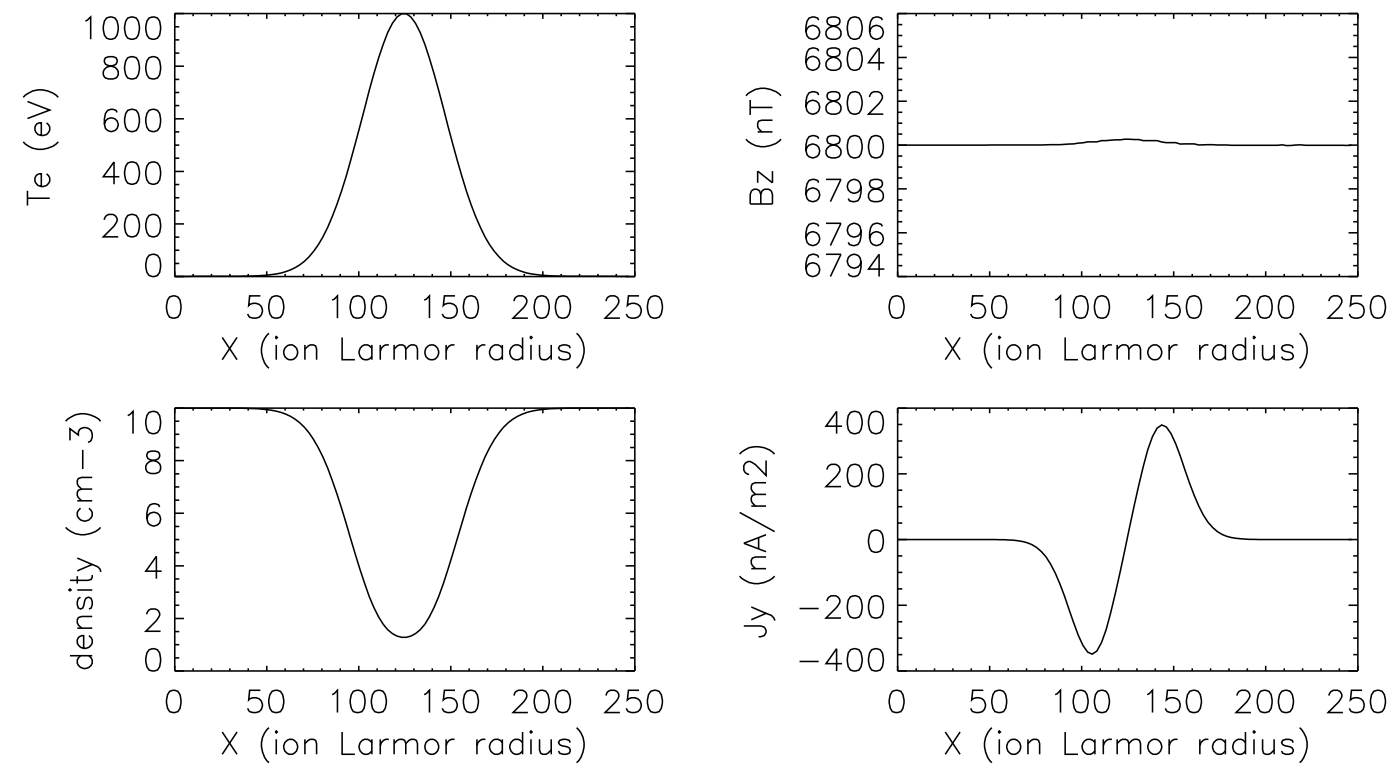

Figure 6: FIG. 6. An example of a deep plasma cavity containing hot electrons $(1 \mathrm{keV})$ surrounded by a cold $(1 \mathrm{eV})$ highly magnetized plasma. See section III.D for details. 